\title{
Supernetworks for Combined Travel Choice Models
}

\author{
Huey-Kuo Chen ${ }^{*}$
}

Department of Civil Engineering, National Central University, Taoyuan 320, Taiwan

\begin{abstract}
A supernetwork is usually defined as an augmented network that consists of a "basic network" for route choice and a "virtual network" for other travel choices. Supernetwork representations are useful pedagogical device to interpret various combined travel choice models as an extension of the fixed demand traffic assignment problem. Based on three proposed criteria, this paper reviews current supernetworks and modifies them as needed for better representation. A nested combined model consisting of four choices subject to variable demand is described with an example. Using supernetwork representations, combined travel choice models have great potential to become more popular than sequential four-step travel demand forecasting, even with feedback. In addition, recently developed quick-precision traffic assignment algorithms could be more efficient than traditional multistage solution algorithms for solving combined travel choice models. The proposed new supernetwork representations can be extended to other network problems in the supply chain network or other economics networks with minor modifications.
\end{abstract}

Keywords: Supernetwork, trip origins, trip distribution, modal choice, traffic assignment.

\section{INTRODUCTION}

Essential for transportation planning, travel demand forecasting contains four travel choices: trip origins or frequency of travel, origin-destination (abbreviated as O-D hereafter), mode, and route (traffic assignment). Traditionally, these four choices are performed as a topdown procedure, shown in Fig. (1).

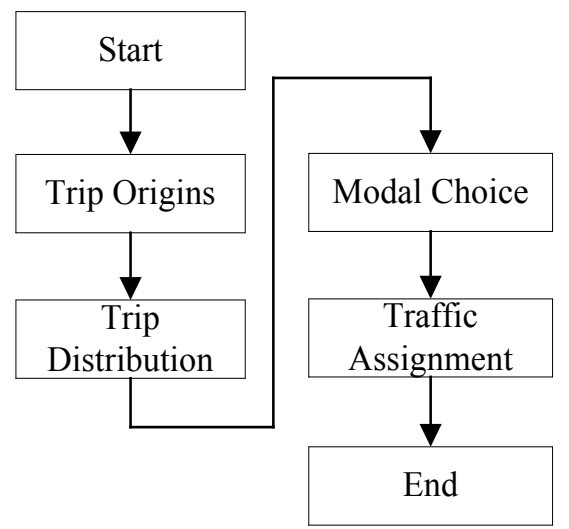

Fig. (1). Sequential travel forecasting in transportation planning.

Among the four travel choice decisions, fixed demand traffic assignment, also known as standard or basic traffic assignment, is recognized as the most fundamental module due to its direct and crucial impact on network congestion. In real applications, traffic assignment is often considered along with one or more other travel decisions. When they are considered within a unified framework, a combined travel choice model (hereafter called the combined model) is formed.

\footnotetext{
*Address correspondence to this author at the Department of Civil Engineering, National Central University, No. 300, Jung-Da Road, Jung-Li City, Taoyuan 320, Taiwan; Tel: +886-3-4227151, Ext. 34115; Fax: +8863-4252960; E-mail: ncutone@ncu.edu.tw
}

Beckmann, McGuire, and Winsten [1] pioneered the original variable demand-route choice model. Sheffi [2] synthesized his contributions on combined models, as well as integrated the findings of other scholars. An extensive historical account and mathematically rigorous synthesis of combined models was prepared by Patriksson [3]. Oppenheim [4] made several theoretical advances to origindestination-mode choice models based on random utility theory. Syntheses and reviews of combined models were elaborately offered by Boyce, LeBlanc and Chon [5], Boyce $[6,7]$ and Boyce and Bar-Gera $[8,9]$. Here we mainly focus on four travel choice dimensions, i.e., trip origins (TO), trip distribution (TD), mode choice (MC) and traffic assignment (TA) assuming travel demand is known and fixed. However, the variable demand may also be considered when needed. By all possible combinations, there are eight nested combined models:

1. Two-step combined models: modal choice/traffic assignment (MC/TA), trip distribution/traffic assignment (TD/TA), and variable demand/traffic assignment (VD/TA);

2. Three-step combined models: TD/MC/TA, TO/MC/ TA and TO/TD/TA;

3. Four-step combined models: TO/TD/MC/TA with fixed demand, TO/TD/MC/TA with variable demand.

Combined models are extensions of the fixed demand traffic assignment problem but are more difficult to understand. However, if combined models can be interpreted as an augmented network, like the fixed demand traffic assignment model is interpreted by the basic network, this difficulty could be lessened. This augmented network is called a supernetwork.

A supernetwork requires the addition of a virtual network to the basic network created from a real physical network. The basic network with real nodes and links is used for route choice, while the virtual network with virtual nodes and links 
reflects one or more other choice dimensions. Naturally, real links must be associated with measured/estimated link costs, and virtual links are characterized by hypothesized cost functions that can appropriately represent the associated travel choice dimensions. Using this type of supernetwork representation, combined models can be addressed as an extension of the fixed demand traffic assignment problem to which all fixed demand traffic assignment algorithms are applicable for solution.

Since the supernetwork representation is a useful pedagogical device and possibly a stimulus for introducing more efficient algorithms for combined models, the structure of currently available virtual networks should be examined and modified if necessary. To examine the appropriateness of supernetwork representations in the literature, we propose three criteria:

1. The supernetwork can address the combined model as an extended fixed demand traffic assignment problem. By virtue of traffic assignment, the corresponding objective function is formed by summing the integral of all link cost functions used in the supernetwork.

2. The supernetwork can be divided into a basic network and a virtual network. The real links in the basic network must be associated with measure/estimated real link cost, while the virtual links embedded in the virtual network must be able to characterize the intended travel choice dimensions. Both userequilibrium (UE) and non-UE conditions can be well represented by the supernetwork by assigning suitable hypothesized cost functions to the virtual links. Note that UE is defined by the Wardrop principles [10], while the non-UE conditions are usually characterized by a multinomial logit function (or logit function in short) [2, p. 364]. In a narrower sense, the term of non-UE is equivalent to "stochastic" UE.

3. The virtual network embedded in a supernetwork requires the addition of as few virtual nodes and links as possible, considering computational efficiency.

Note that our purpose is pedagogy, mainly to explain the relationship of our supernetwork representation to the standard ones. Therefore graphs are used for ease of comparison. This paper is organized as follows. In Section 2, we review the supernetworks associated with two-step combined models. Section 3 extends the discussion to threestep combined model. Section 4 elaborates four-step combined models. Section 5 formulates the combined $\mathrm{TO} / \mathrm{TD} / \mathrm{MC} / \mathrm{TA}$ problem with variable demand as an extended traffic assignment problem and derives its optimality conditions. Section 6 further demonstrates with a numerical example the $\mathrm{TO} / \mathrm{TD} / \mathrm{MC} / \mathrm{TA}$ problem with variable demand. Section 7 concludes the paper.

\section{SUPERNETWORKS FOR TWO-STEP COMBINED MODELS}

\subsection{Combined VD/TA Model}

The variable demand traffic assignment problem was first formulated as an optimization model by Beckmann, McGuire, and Winsten [1]. Gartner [11] introduced two supernetworks, called zero cost overflow formulation and excess-demand network representation, for converting the variable demand traffic assignment problem into an equivalent fixed demand traffic assignment. The two supernetworks for the variable demand traffic assignment problem are redrawn in Fig. (2a, b).

In the figures, symbols $t_{r r^{\prime}}, t_{s r^{\prime}}, q^{r s}, e^{r s}, D_{r s}^{-1}(\cdot), W_{r s}\left(e^{r s}\right)$ denote, travel time for link $r r$ ', travel time for link $s r^{\prime}$, trip rate between O-D pair $(r, s)$, excess demand between O-D pair $(r, s)$, inverse demand function between O-D pair $(r, s)$, and the augment-complementing function of the inverse demand, respectively. By virtue of traffic assignment, the objective function for the supernetwork in Fig. (2a) can be constructed as follows:

$$
\begin{aligned}
z & =z \text { (basic network })+z \text { (virtual network }) \\
& =z \text { (basic network })+\sum_{r r^{\prime}} \int_{r^{\prime}}^{x_{r r^{\prime}}} t^{\prime}(\omega) d \omega+\sum_{r s} \int_{0}^{x_{s^{\prime}}} t_{s r^{\prime}}(\omega) d \omega \\
& =z \text { (basic network })-\sum_{r s} \int_{0}^{q^{q_{s}}} D_{r s}^{-1}(\omega) d \omega
\end{aligned}
$$

where $x_{r r^{\prime}}, x_{s r^{\prime}}$ denote flows on link $r r^{\prime}$ and link $s r^{\prime}$, respectively. Interestingly, the resulting objective function in Equation 1 is identical to the objective function that corresponds to the supernetwork presented in Fig. (2b). However, due to the fewer nodes and links required for the virtual network, the supernetwork shown in Fig. (2b) is preferable to Fig. (2a) for practical use.

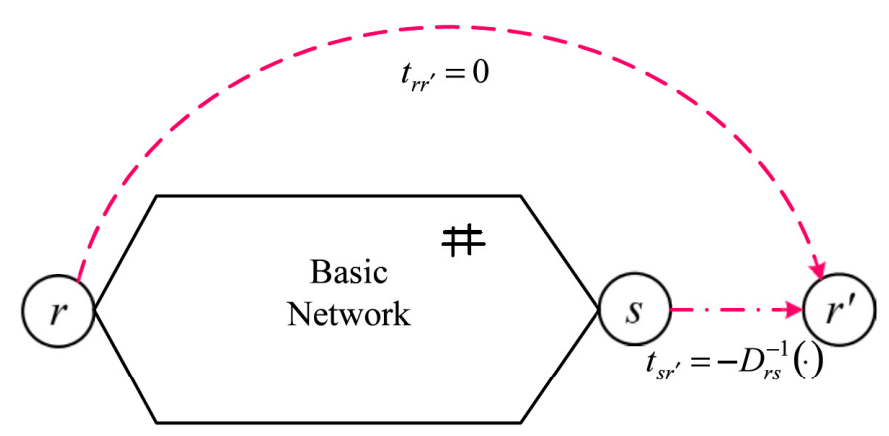

(a)

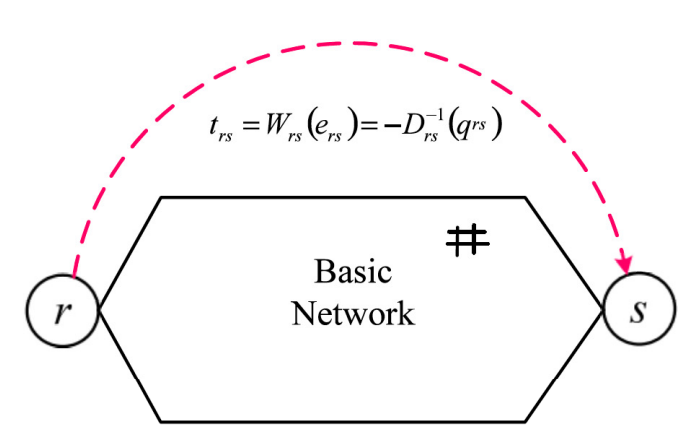

(b)

Fig. (2). Gartner's supernetwork representation. (a) Zero-cost overflow formulation; (b) Excess-demand supernetwork (Source: Gartner [11]; Sheffi [2], p. 147 and p. 150). 


\subsection{Combined TD/TA Model}

The first trip distribution/traffic assignment model is due to Evans [12]. Sheffi [2] presents a supernetwork for this problem. It is reproduced in Fig. (3).

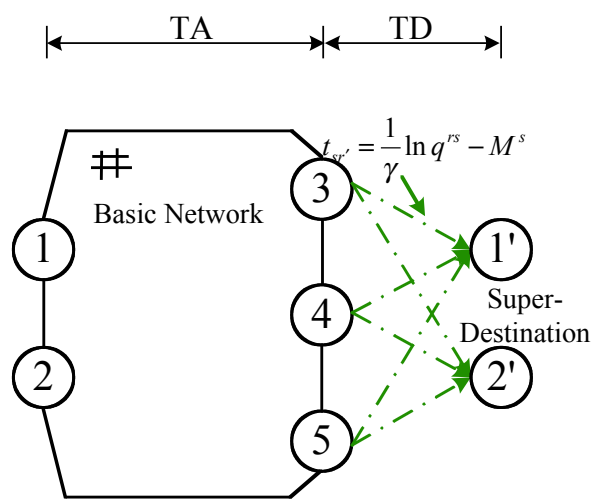

Fig. (3). Sheffi's combined TD/TA network representation (Source: Sheffi [2], pp. 169-170).

By virtue of traffic assignment, the objective function for the supernetwork in Fig. (3) can be formulated as

$$
\begin{aligned}
z & =z(\text { basic network })+z(\text { virtual network }) \\
& =z(\text { basic network })+\sum_{s r^{\prime}} \int_{0}^{x_{s r^{\prime}}} t_{s r^{\prime}}(\omega) d \omega \\
& =z(\text { basic network })+\sum_{r s} \int_{0}^{q^{r s}}\left(\frac{1}{\gamma} \ln \omega-M^{s}\right) d \omega \\
& =z(\text { basic network })+\frac{1}{\gamma} \sum_{r s} q^{r s} \ln q^{r s}-\sum_{r s} M^{s} q^{r s}
\end{aligned}
$$

where $\gamma, M^{s}$ are the parameter of the logit model and attraction measure associated with destination $s$. Note that with the given cost function in the virtual network, this supernetwork is essentially a non-UE type assignment problem. However, if the virtual links are assumed with zero cost, then the UE-type assignment problem emerges. Since Sheffi's supernetwork satisfies the three criteria, no better supernetwork representation exists.

\subsection{Combined MC/TA Model}

Sheffi [2] also presents two supernetworks for the combined MC/TA problem. The first supernetwork, shown in Fig. (4a), corresponds to a UE-type assignment.
In Fig. (4a), each origin and destination is decomposed by modes and connected by virtual links. All virtual links $r \rightarrow r^{\prime}, r \rightarrow \hat{r}, s^{\prime} \rightarrow s, \hat{s} \rightarrow s$ have zero costs. By virtue of traffic assignment, the objective function for the supernetwork in Fig. (4a) can be formulated as follows:

$$
\begin{aligned}
z= & z \text { (basic network })+z(\text { virtual network }) \\
= & z \text { (basic network })+\sum_{r r^{\prime}} \int_{0}^{x_{r^{\prime}}} t_{r r^{\prime}}(\omega) d \omega+\sum_{r \hat{r}} \int_{0}^{x_{r^{\prime}}} t_{r \hat{r}}(\omega) d \omega \\
& +\sum_{s^{\prime} s} \int_{0}^{x_{s s} s} t_{s^{\prime} s}(\omega) d \omega+\sum_{\hat{s s}} \int_{0}^{x_{\hat{s} s}} t_{\hat{s s}}(\omega) d \omega \\
= & z \text { (basic network) }
\end{aligned}
$$

As shown in Equation 3a, the objective function corresponding to the supernetwork presented in Fig. (4a) can be simplified as the objective function for the basic network. In other words, the addition of four zero virtual links to the basic network is not required for this UE-type assignment. Therefore, we instead propose the original basic network in Fig. (4b) for use, which indeed does not require the addition of virtual links.

Sheffi's [2] second supernetwork for the MC/TA problem, shown in Fig. (5a), is associated with the non-UE transit assignment model.

In Fig. (5a), the travel time over equivalent transit links, $\hat{t}_{r s}$ is given by the performance function shown below:

$$
\hat{t}_{r s}\left(\hat{q}^{r s}, \mathbf{x}, \hat{\mathbf{x}}\right)=\frac{1}{\theta} \ln \frac{\hat{q}^{r s}}{\bar{q}^{r s}-\hat{q}^{r s}}+\Psi_{r s}+\hat{u}^{r s}(\mathbf{x}, \hat{\mathbf{x}}) \quad \forall r, s
$$

where $\hat{u}^{r s}(\mathbf{x}, \hat{\mathbf{x}})$ is the minimum travel time over the transit network and is defined as the function of automobile flows $\mathbf{x}$ and transit flows $\hat{\mathbf{x}}$. The symbols $\bar{q}^{r s}, \hat{q}^{r s}, \Psi_{r s}, \theta$ denote, total O-D trip rate, transit O-D trip rate, automobile preference parameter, and logit model parameter associated with each O-D pair, respectively. In this paper the notations of travel time, $t$, and travel cost, $c$, are used interchangeably. Note that the assumed performance function in Equation $3 \mathrm{~b}$ is an artificial function, and has no intuitive meaning from the viewpoint of real transit links (or something to this effect).

By virtue of traffic assignment, the objective function corresponding to the supernetwork shown in Fig. (5a) can be written as follows:

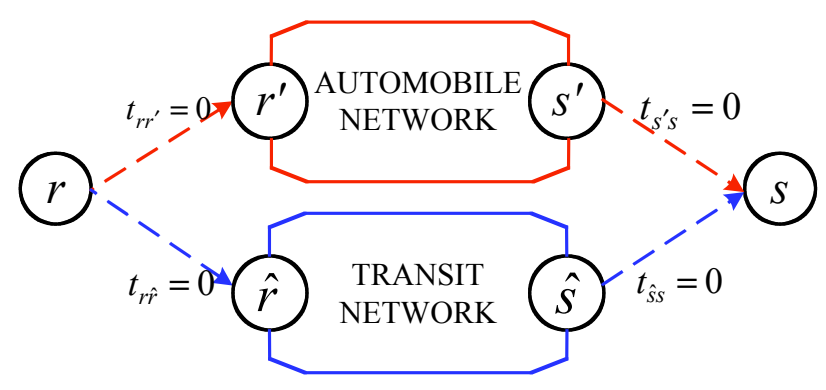

(a) MC/TA(UE)

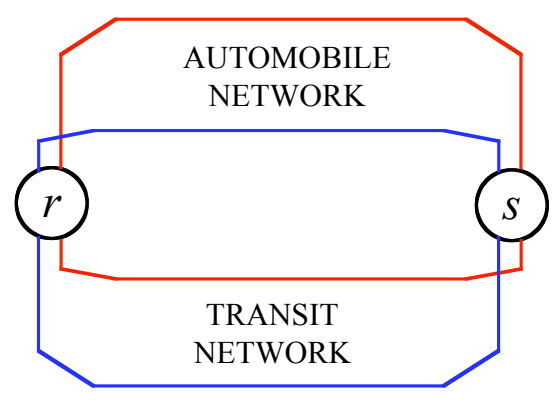

(b) $\mathrm{MC} / \mathrm{TA}(\mathrm{UE})$

Fig. (4). Sheffi's supernetwork representation for MC/TA problem (UE) ([Source: Sheffi [2], p. 234). 


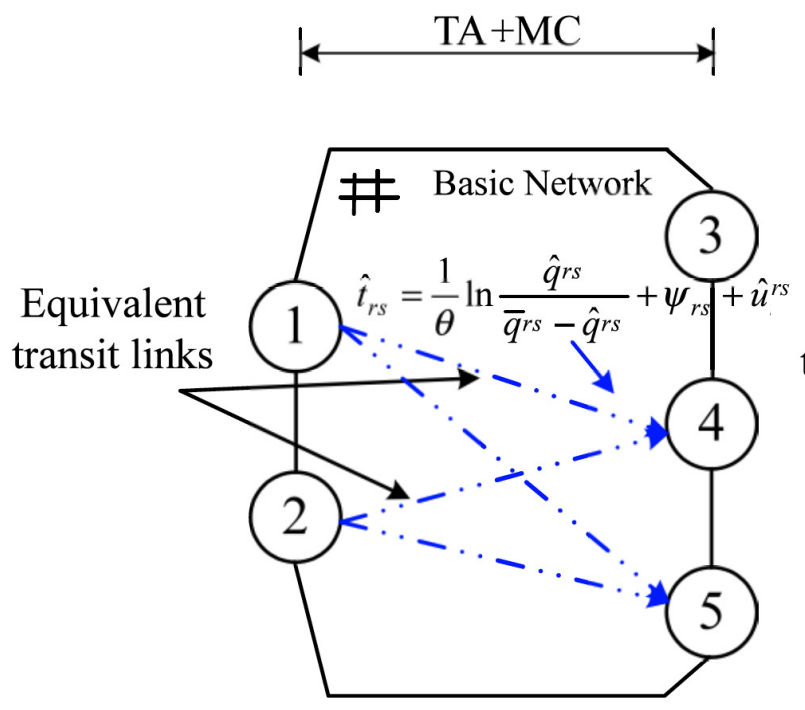

(a) $\mathrm{MC} / \mathrm{TA}$ (non-UE)

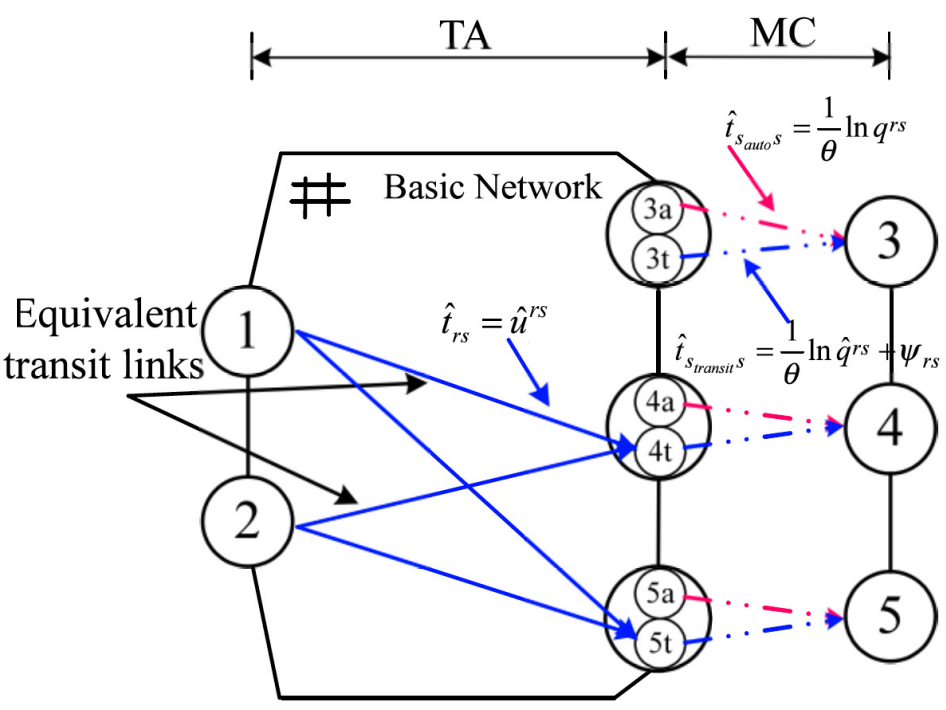

(b) $\mathrm{MC} / \mathrm{TA}$ (non-UE)

Fig. (5). (a) Sheffi's supernetwork Rrepresentation for MC/TA problem (Non-UE) (Source: Sheffi [2], p. 243); (b) Proposed combined $\mathrm{MC} / \mathrm{TA}$ supernetwork representation.

$$
\begin{aligned}
z & =\sum_{a} \int^{x_{a}} t_{a}(\omega) d \omega+\sum_{r s} \int_{0}^{\hat{q}^{r s}}\left(\frac{1}{\theta} \ln \frac{\hat{q}^{r s}}{\bar{q}^{r s}-\hat{q}^{r s}}+\Psi_{r s}+\hat{u}^{r s}(\mathbf{x}, \hat{\mathbf{x}})\right)(\omega) d \omega \\
& =\sum_{a} \int^{x_{a}} t_{a}(\omega) d \omega+\sum_{r s} \int_{0}^{\hat{q}^{s s}} \hat{u}^{r s}(\mathbf{x}, \hat{\mathbf{x}}) d \omega+\sum_{r s} \int_{0}^{\hat{q}^{s}}\left(\frac{1}{\theta} \ln \frac{\hat{q}^{r s}}{\bar{q}^{r s}-\hat{q}^{r s}}+\Psi_{r s}\right) d \omega \\
& =z \text { (basic network })+\sum_{r s} \int_{0}^{\hat{q}^{s}}\left(\frac{1}{\theta} \ln \frac{\hat{q}^{r s}}{\bar{q}^{r s}-\hat{q}^{r s}}+\Psi_{r s}\right) d \omega \\
& =z \text { (basic network })+\sum_{r s} \int_{0}^{q^{s s}}\left(\frac{1}{\theta} \ln \omega\right) d \omega+\sum_{r s} \int_{0}^{\hat{q}^{s s}}\left(\frac{1}{\theta} \ln \omega+\Psi_{r s}\right) d \omega \\
& =z \text { (basic network })+z(\text { virtual network })
\end{aligned}
$$

As shown in Equation 3c, the real link cost for transit mode can be separated from the assumed performance function and then combined with the real link cost for automobile mode to form the objective function for the basic network; after separation, the remaining items correspond to the virtual network. Based on this discussion, the supernetwork representation in Fig. (5a) for the MC/TA problem can be modified as shown in Fig. (5b). The modified supernetwork shown in Fig. (5b) is easier to understand than Sheffi's [2] supernetwork shown in Fig. (5a), as the basic network is purely represented by real link cost functions and virtual network is assumed with link cost functions to characterize modal choice with logit formula.

\section{SUPERNETWORK FOR THREE-STEP COMBINED MODEL}

Theoretically, three three-step combined models are possible: TO/TD/TA, TO/MC/TA, and TD/MC/TA. However, Sheffi [2] includes only the supernetwork for the TD/MC/TA combined model, shown in Fig. (6a):

By virtue of traffic assignment, the objective function corresponding to the supernetwork shown in Fig. (6a) can be written as follows:

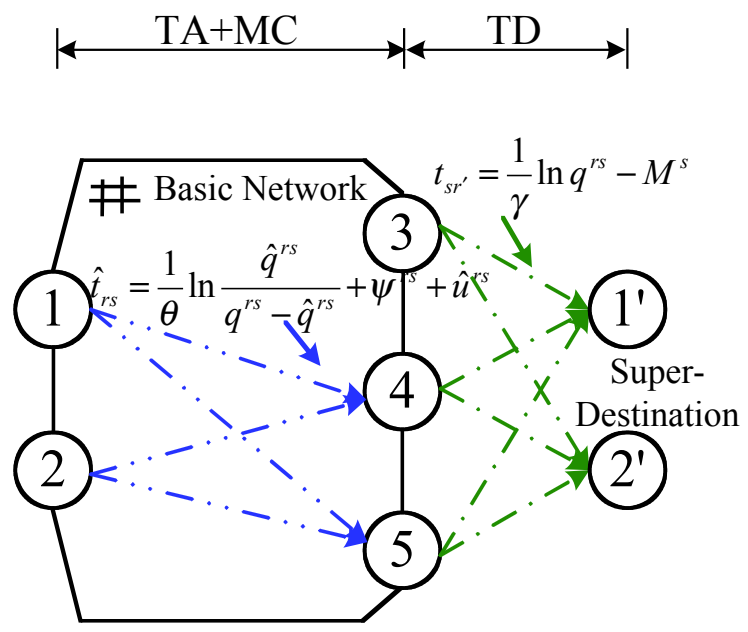

(a)

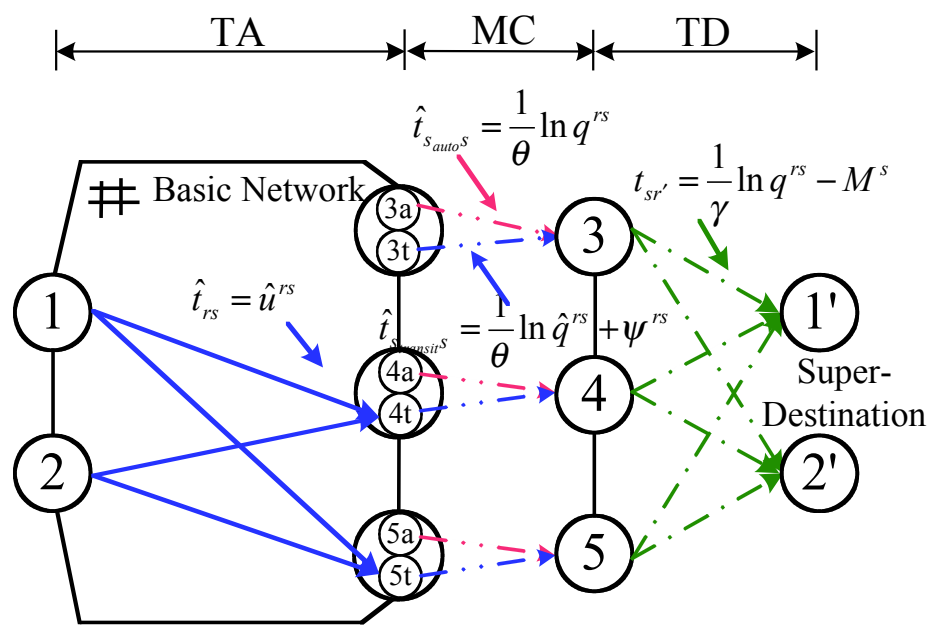

(b)

Fig. (6). (a) Sheffi's combined TD/MC/TA supernetwork representation (Source: Sheffi [2], p. 248); (b) Proposed combined TD/MC/TA supernetwork representation. 


$$
\begin{aligned}
& z=\# a_{a}{ }^{x_{a}} t_{a}(!) d !+\#{ }_{r s} \quad \hat{q}^{n} \quad\left(-\ln \frac{\hat{q}^{r s}}{\bar{q}^{r s}-\hat{q}^{r s}}+\%_{r s}+\hat{u}^{r s}(\mathbf{x}, \hat{\mathbf{x}})_{+}\right)(!) d !
\end{aligned}
$$

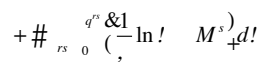

$$
\begin{aligned}
& =\#{ }_{a}{ }^{x_{a}} t_{a}(!) d !+\#{ }_{r s}{ }_{0}^{\hat{q}^{r s}} \hat{u}^{r s}(\mathbf{x}, \hat{\mathbf{x}}) d !+\#{ }_{r s} \quad \hat{q}_{0}^{r s}\left(-\ln \frac{\hat{q}^{r s}}{\bar{q}^{r s}-\hat{q}^{r s}}+\%_{r s}\right) d ! \\
& +\#_{r s} \quad q_{0}^{n:}\left(-\ln ! \quad M^{s}\right) d ! \\
& =z(\text { basic network })+\#{ }_{r s} \quad \hat{q}_{0}^{n^{n}} \& 11
\end{aligned}
$$

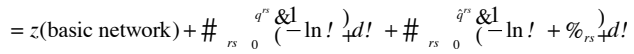

$$
\begin{aligned}
& +-\#_{r s} q^{r s} \ln q^{r s} \#_{r s} M^{s} q^{r s} \\
& =z \text { (basic network })+z(\text { virtual network })
\end{aligned}
$$

The symbols $\gamma$ and $M^{s}$ denote respectively the logit model associated with mode choice and attractiveness at destination $s$. From the result shown in Equation 4, it is clear that the objective function can be divided into two parts: a basic network and a virtual network. Note that the virtual network can be further divided into two subclasses, i.e., mode choice and trip distribution. To better represent this resulting objective function, a new supernetwork representation is proposed in Fig. (6b). Clearly, three steps of travel choices have been orderly represented in the new supernetwork.

With the aforementioned discussion and the new supernetworks proposed for the three-step combined models, the supernetworks for the four-step combined models can be easily constructed by analogy.

\section{SUPERNETWORK FOR FOUR-STEP COMBINED MODEL}

\subsection{Supernetwork for Four-Step Combined Models with Fixed Demand}

The four-step combined model with fixed demand, i.e., $\mathrm{TO} / \mathrm{TD} / \mathrm{MC} / \mathrm{TA}$, is one of the most complex cases among combined models. Sheffi's [2] supernetwork covering four steps of travel choices is redrawn in Fig. (7a):

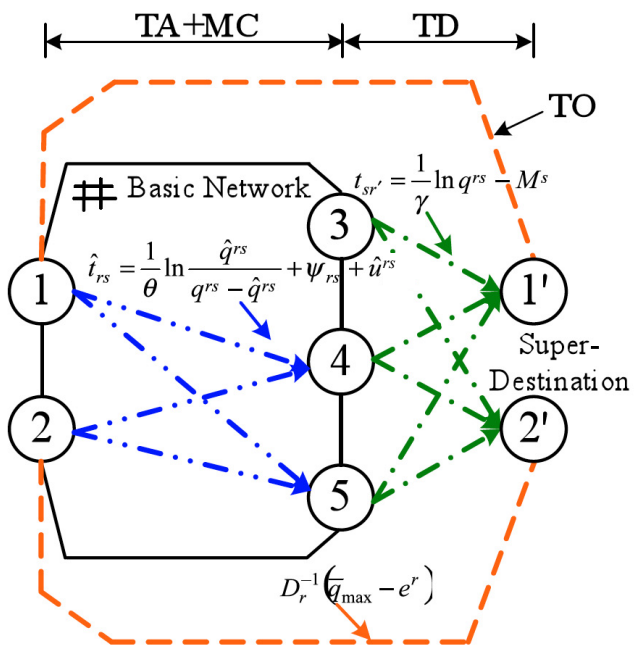

(a)

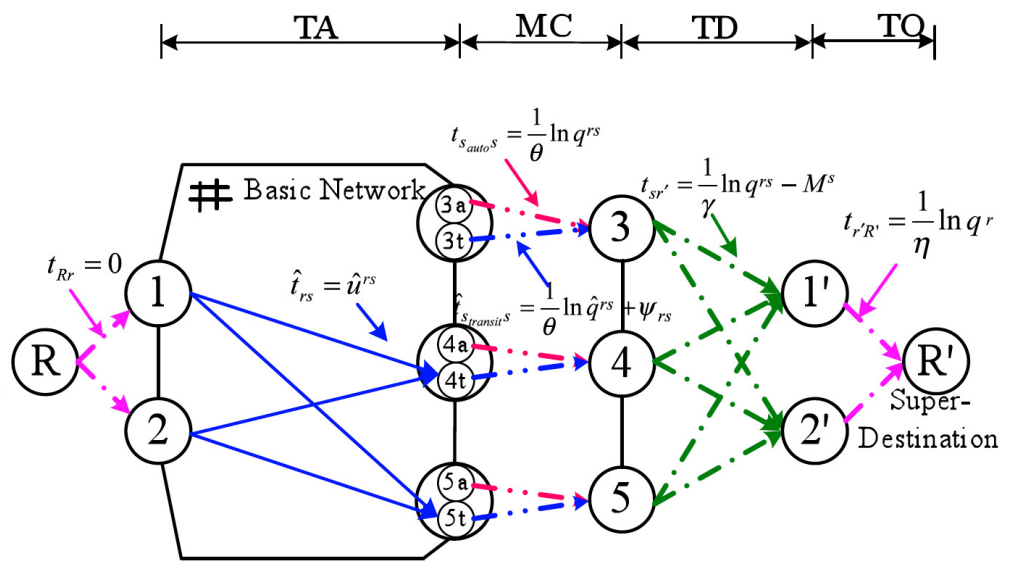

(b)

Fig. (7). (a) Sheffi's supernetwork for TO/TD/MC/TA combined model (Source: Sheffi [2], p. 253). (b) Proposed supernetwork for combined TO/TD/MC/TA model with Fixed Demand. 


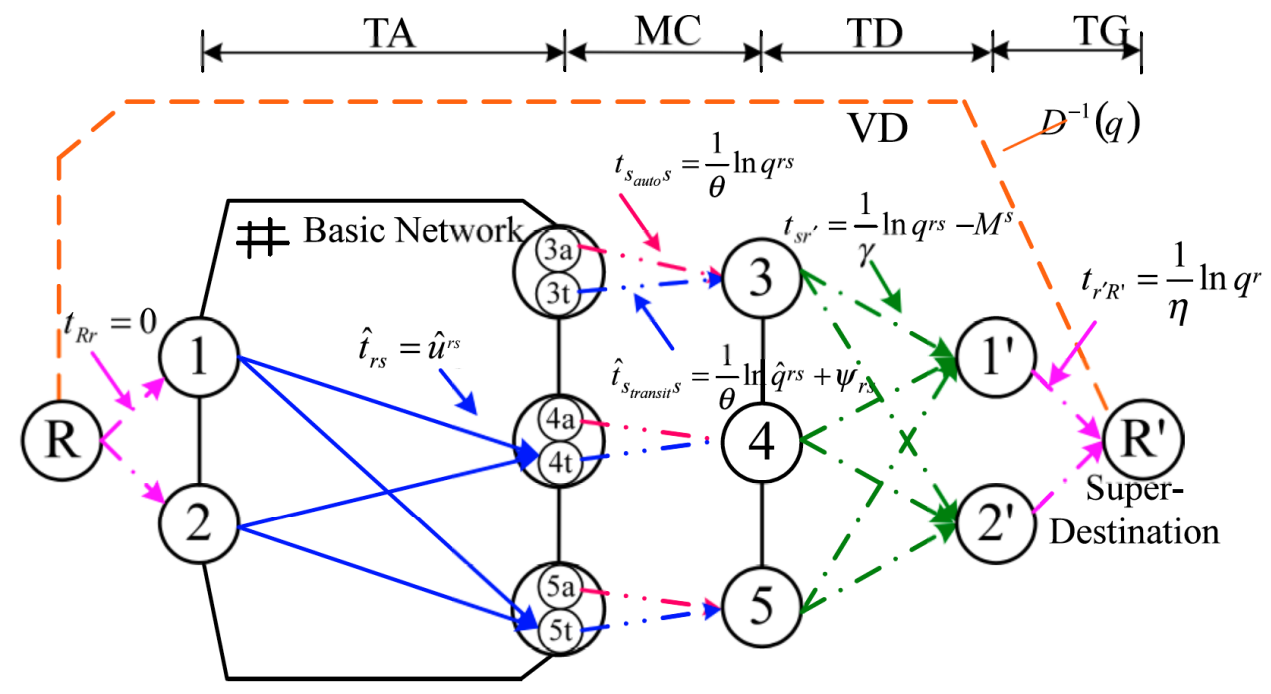

Fig. (7c). Proposed supernetwork for combined TO/TD/MC/TA model with variable demand.

\section{Variable Demand}

If variable demand is incorporated into the four-step combined model, the corresponding supernetwork representation can be drawn in Fig. (7c) by analogy:

The supernetwork representation proposed in Fig. (7c) implies the following objective function.

$$
\begin{aligned}
z= & \sum_{a} \int^{x_{a}} t_{a}(\omega) d \omega+\sum_{r s} \int_{0}^{q^{s s}}\left(\frac{1}{\theta} \ln \omega\right) d \omega+\sum_{r s} \int_{0}^{\hat{q}^{r s}}\left(\frac{1}{\theta} \ln \omega+\Psi_{r s}\right) d \omega \\
& +\frac{1}{\gamma} \sum_{r s} q^{r s} \ln q^{r s}-\sum_{r s} M^{s} q^{r s}+\frac{1}{\eta} \sum_{r} q^{r} \ln q^{r}-\int_{0}^{q} D^{-1}(\omega) d \omega \\
= & z \text { (basic network })+z \text { (virtual network })
\end{aligned}
$$

Note that the flow over the excess link $R \rightarrow R$ ' denotes the flow of "nontravelers" in the network being studied.

\section{MODEL FORMULATION AND OPTIMALITY CONDITIONS FOR THE COMBINED TO/TD/MC/TA PROBLEM WITH VARIABLE DEMAND}

In the following sections, we show that the combined TO/TD/MC/TA problem with variable demand can be formulated as an extended traffic assignment model with the help of supernetwork representation and derive its optimality conditions, which must be consistent with the generalized Wardrop principle.

\subsection{Formulating the Combined TO/TD/MC/TA Problem with Variable Demand by Supernetwork Representation}

As the four-step combined TO/TD/MC/TA problem with variable demand is an extension of the traffic assignment problem, it must consist of an objective function and a feasible region that includes three types of constraints (i.e., flow conservation, non-negative and definitional constraints). With the help of supernetwork representation, the mathematical model corresponding to the four-step combined TO/TD/MC/TA problem with variable demand can be formulated as follows:

$$
\begin{aligned}
& \min _{\left(x, q_{m}^{\prime \prime}, q^{s}, q^{\prime}\right) \in \Omega} z=\sum_{m} \sum_{l} \int_{0}^{x_{m l}} c_{m l}(\omega) d \omega+\sum_{r s} \sum_{m} \int_{0}^{q_{m}^{s}} c_{m}^{r s}(\omega) d \omega \\
& +\sum_{s r^{\prime}} \int_{0}^{q^{q^{\prime}}} c_{s r^{\prime}}(\omega) d \omega+\sum_{r^{\prime} R^{\prime}} \int_{0}^{q^{r}} c_{r^{\prime} R^{\prime}}(\omega) d \omega-\int_{0}^{q} D^{-1}(\omega) d \omega
\end{aligned}
$$

where the feasible region $\Omega$ is defined by the following constraints.

Flow Conservation Constraints:

$$
\begin{array}{ll}
\sum_{p} f_{m p}^{r s}=q_{m}^{r s} & \forall r, s, m \quad\left(\mu_{m}^{r s}\right) \\
\sum_{m} q_{m}^{r s}=q^{r s} & \forall r, s \quad\left(\mu^{r s}\right) \\
\sum_{s} q^{r s}=q^{r} & \forall r \quad\left(\mu^{r}\right) \\
\sum_{r} q^{r}=q & (\mu) \\
q+e=\bar{q}_{\max } & \left(\mu_{\max }\right)
\end{array}
$$

Non-negativity Constraints:

$$
f_{m p}^{r s} \geq 0 \quad \forall r, s, m, p
$$

Definitional Constraints:

$$
\begin{aligned}
& \sum_{m} x_{m l}=\sum_{r} \sum_{s} \sum_{m} \sum_{p} f_{m p}^{r s} \delta_{m l p}^{r s} \quad \forall l \\
& \sum_{m} c_{m p}^{r s}=\sum_{m} \sum_{l} c_{m l} \delta_{m l p}^{r s} \quad \forall r, s, p
\end{aligned}
$$

where

$\bar{q}_{\max }$ is the upper limit of total OD trip rate from the entire area;

$q$ is the total OD trip rate from the entire area;

$q^{r}$ is the total OD trip rate from origin $r$;

$q^{r s}$ is the total OD trip rate between origin $r$ and destination $s$;

$q_{m}^{s}$ is the total flow by mode $m$ arriving destination $s$;

$q_{m}^{r s}$ is the total flow by mode $m$ between origin $r$ and destination $s$;

$e$ is the excess flow associated with the entire area; 
$D^{-1}(q)$ is the inverse demand function; equivalent to the excess demand function $E(e)$; and

$c_{m l}$ is the travel cost function associated with link $l$ and mode $m$.

Equation 6a defines the objective function by summing the integrals of link travel costs for all links, including real links for traffic assignment and virtual links for modal choice, trip distribution, trip origin as well as variable demand. Equation $6 \mathrm{~b}$ conserves flows for each O-D pair by mode $m$. Eq. $6 \mathrm{c}$ conserves flows for each O-D pair. Equation $6 \mathrm{~d}$ conserves flows for each origin $r$. Equation 6e conserves flows for the entire area. Equation $6 \mathrm{f}$ sets the upper limit of total traffic demand for the entire area. Equation $6 \mathrm{~g}$ requires path flow associated with each mode and route be negative. Equations $6 \mathrm{~h}$ and $6 \mathrm{i}$ are definitional constraints.

The link travel costs of the virtual links are assumed based mainly on the logit formula, as follows:

$c_{m}^{r s}=\frac{1}{\theta} \ln q_{m}^{r s}-M_{m}^{s}$

$c_{s r^{\prime}}=\frac{1}{\gamma} \ln q^{r s}-M^{s}$

$c_{r^{\prime} R^{\prime}}=\frac{1}{\eta} \ln q^{r}$

$c_{R R^{\prime}}=D^{-1}(q)$

where

$\theta, \gamma, \eta$ are the logit function dispersion parameters associated with modal choice, trip distribution and trip generation, respectively;

$M^{s}$ is the attractiveness measure associated with destination $s$; and

$M_{m}^{s}$ is the preference parameter associated with destination $s$ and mode $m$.

Then, the above mathematical model can be rewritten as follows:

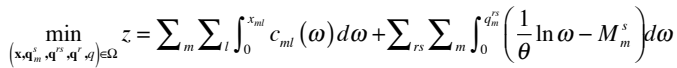

$$
\begin{aligned}
& +\sum_{r s} \int_{0}^{q^{s}}\left(\frac{1}{\gamma} \ln \omega-M^{s}\right) d \omega+\sum_{r} \int_{0}^{q^{q}}\left(\frac{1}{\eta} \ln \omega\right) d \omega-\int_{0}^{q} D^{-1}(\omega) d \omega
\end{aligned}
$$

To show that this model can result in the intended travelers' behavior, we derive its optimality conditions in the following section.

\subsection{Optimality Conditions}

Let dual variables associated with Equations $6 \mathrm{~b}$ through 6f be $\mu_{m}^{r s}, \mu^{r s}, \mu^{r}, \mu$ and $\mu_{\max }$, respectively. The corresponding Lagrangian function associated with the objective function in Equation $8 \mathrm{a}$ can be expressed as follows:

$$
\begin{aligned}
& \mathscr{C}\left(\mathbf{x}, \mathbf{q}_{n}^{r s}, \mathbf{q}^{r s}, \mathbf{q}^{r}, q, \mathbf{u}_{m}^{s s}, \mathbf{u}^{r s}, \boldsymbol{\mu}^{r}, \mu\right) \\
& =\sum_{m} \sum_{I} \int_{0}^{x_{m}} c_{m 1}(\omega) d \omega+\sum_{r s} \sum_{m} \int_{0}^{g^{m}}\left(\frac{1}{\theta} \ln \omega-M_{m}^{s}\right) d \omega \\
& +\sum_{r s} \int_{0}^{q^{q}}\left(\frac{1}{\gamma} \ln \omega-M^{*}\right) d \omega+\sum \int_{0}^{q}\left(\frac{1}{\eta} \ln \omega\right) d \omega-\int_{0}^{q} D^{-1}(\omega) d \omega \\
& +\sum_{r s} \sum_{m} \mu_{m}^{r s}\left(q_{m}^{s s}-\sum_{p} f_{m p}^{s s}\right)+\sum_{r s} \mu^{r s}\left(q^{r s}-\sum_{m} q_{m}^{r s}\right)+\sum_{r} \mu^{r}\left(q^{r}-\sum_{s} q^{r s}\right) \\
& +\mu\left(q-\sum_{r} q^{r}\right)+\mu_{\max }\left(\bar{q}_{\max }-q\right)
\end{aligned}
$$

The corresponding optimality conditions can be obtained by taking derivatives with respect to all primal and dual decision variables. Since the optimality conditions corresponding to the dual decision variables yield the original constraints, we only need to discuss the optimality conditions with respect to the primal decision variables, as follows:

(1) The necessary conditions associated with decision variables $q$ (denoting variable demand) are:

$\frac{\partial \mathscr{Q}}{\partial q} \geq 0$

$q \frac{\partial \mathscr{Q}}{\partial q}=0$

Since $\frac{\partial \mathscr{Q}}{\partial q}=\mu-\mu_{\max }-D^{-1}(q)$, substituting it into Equations $9 \mathrm{a}$ and $9 \mathrm{~b}$ yields

$\frac{\partial \mathscr{Q}}{\partial q}=\mu-\mu_{\max }-D^{-1}(q) \geq 0$

$q \frac{\partial \mathscr{Q}}{\partial q}=q\left(\mu-\mu_{\max }-D^{-1}(q)\right)=0$

Conditions $9 \mathrm{c}$ and $9 \mathrm{~d}$ have similar interpretations. If the total origins are positive, i.e., $q>0$, then, $\mu_{\max }=0$ and for Equation 9d to hold, Equation 9c must hold as an equality. In other words,

$\mu=D^{-1}(q)$

Both sides of this equation can be inverted to obtain

$D(\mu)=q$

Thus, if $q>0$, it must be given by the demand function. If, however, $q=0$, then from Equation $9 \mathrm{c}$ we find that

$\mu-\mu_{\max } \geq D^{-1}(q)$

meaning that the travel time may be too high to induce any travelers in the study area.

(2) The necessary conditions associated with decision variables $q^{r}$ (denoting trip origins) are:

$\frac{\partial \mathscr{Q}}{\partial q^{r}} \geq 0$

$q^{r} \frac{\partial \mathscr{Q}}{\partial q^{r}}=0$ 


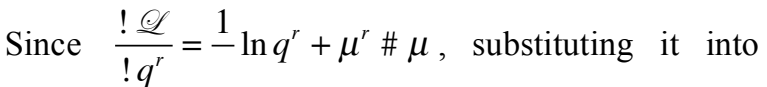
Equations $10 \mathrm{a}$ and $10 \mathrm{~b}$ yields

$\frac{! \mathscr{Q}}{! q^{r}}=\frac{1}{-} \ln q^{r}+\mu^{r} \# \mu \quad 0$

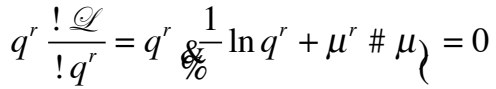

Conditions $10 \mathrm{c}$ and $10 \mathrm{~d}$ have similar interpretations. If the trip origins are positive, i.e., $q^{r}>0$, then, for Equation 10d to hold, Equation 10c must hold as an equality. In other words,

$\frac{1}{\eta} \ln q^{r}+\mu^{r}-\mu=0$

Both sides of this equation can be inverted to obtain

$\ln q^{r}=-\eta\left(\mu^{r}-\mu\right)$

This can be rewritten as

$q^{r}=e^{-\eta\left(u^{r}-\mu\right)}$

Summing over $r$, we have

$q=\sum_{r} e^{-\eta\left(u^{r}-\mu\right)}$

Dividing Equation 10e by Equation 10f results in a logit function:

$\frac{q^{r}}{q}=\frac{e^{-\eta\left(u^{r}-\mu\right)}}{\sum_{r^{\prime}} e^{-\eta\left(u^{r^{\prime}}-\mu\right)}}=\frac{e^{-\eta u^{r}}}{\sum_{r^{\prime}} e^{-\eta u^{r^{\prime}}}}$

(3) The necessary conditions associated with decision variables $q^{r s}$ (denoting total O-D demand) are:

$\frac{\partial \mathscr{\ell}}{\partial q^{r s}} \geq 0$

$q^{r s} \frac{\partial \mathscr{Q}}{\partial q^{r s}}=0$

By derivation, $\frac{\partial \mathscr{Q}}{\partial q^{r s}}=\frac{1}{\gamma} \ln q^{r s}-M^{s}+u^{r s}-\mu^{r}$. Since $\ln q^{r s}$ is not defined for $q^{r s}=0$, the inequality $q^{r s}>0$ must hold. By Equation $11 \mathrm{~b}, \frac{\partial \mathscr{Q}}{\partial q^{r s}}=0$, i.e.,

$\frac{\partial \mathscr{Q}}{\partial q^{r s}}=\frac{1}{\gamma} \ln q^{r s}-M^{s}+\mu^{r s}-\mu^{r}=0$

By manipulation, this results in

$\ln q^{r s}=-\gamma\left(u^{r s}-M^{s}-\mu^{r}\right)$

This can be rewritten as

$q^{r s}=e^{-\gamma\left(\mu^{r s}-M^{s}-\mu^{r}\right)}$

Summing over s', we have $q^{r}=\sum_{s^{\prime}} e^{-\gamma\left(\mu^{r s^{\prime}}-M^{\left.s^{\prime}-\mu^{r}\right)}\right.}$

Dividing Equation 11e by Equation $11 \mathrm{f}$ results in a logit function:

$\frac{q^{r s}}{\bar{q}^{r}}=\frac{e^{-\gamma\left(\mu^{s s}-M^{s}-\mu^{r}\right)}}{\sum_{s^{\prime}} e^{-\gamma\left(\mu^{s^{s}}-M^{s^{s}}-\mu^{r}\right)}}=\frac{e^{-\gamma\left(\mu^{s^{s}}-M^{s}\right)}}{\sum_{s^{\prime}} e^{-\gamma\left(\mu^{s^{s}}-M^{s^{\prime}}\right)}}$

(4) The necessary conditions associated with decision variables $q_{m}^{r s}$ (denoting O-D demand by mode) are:

$\frac{\partial \mathscr{Q}}{\partial q_{m}^{r s}} \geq 0$

$q_{m}^{r s} \frac{\partial \mathscr{Q}}{\partial q_{m}^{r s}}=0$

By derivation, $\frac{\partial \mathscr{Q}}{\partial q_{m}^{r s}}=\frac{1}{\theta} \ln q_{m}^{r s}-M_{m}^{s}+\mu_{m}^{r s}-\mu^{r s}$. Since

$\ln q_{m}^{r s}$ is not defined when $q_{m}^{r s}=0, q_{m}^{r s}>0$ must hold. Equation $12 \mathrm{~b}$ yields $\frac{\partial \mathscr{Q}}{\partial q_{m}^{r s}}=0$, i.e.,

$\frac{\partial \mathscr{Q}}{\partial q_{m}^{r s}}=\frac{1}{\theta} \ln q_{m}^{r s}-M_{m}^{s}+u_{m}^{r s}-\mu^{r s}=0$

With manipulation, this results in

$\ln q_{m}^{r s}=-\theta\left(u_{m}^{r s}-M_{m}^{s}-\mu\right)$

which can be rewritten as

$q_{m}^{r s}=e^{-\theta\left(u_{m}^{r s}-M_{m}^{s}-\mu^{r s}\right)}$

Summing over $m$ ', we have

$\sum_{m^{\prime}} q_{m^{\prime}}^{r s}=\sum_{m^{\prime}} e^{-\theta\left(u_{m^{\prime}}^{r s}-M_{m^{\prime}}^{s}-\mu^{r s}\right)}$

Dividing Equation $12 \mathrm{e}$ by Equation $12 \mathrm{f}$ results in a logit formula:

$\frac{q_{m}^{r s}}{\sum_{m^{\prime}} q_{m^{\prime}}^{r s}}=\frac{e^{-\theta\left(\mu_{m}^{r s}-M_{m}^{s}-\mu^{r s}\right)}}{\sum_{m^{\prime}} e^{-\theta\left(\mu_{m^{\prime}}^{r s}-M_{m^{\prime}}^{s}-\mu^{r s}\right)}}=\frac{e^{-\theta\left(\mu_{m}^{r s}-M_{m}^{s}\right)}}{\sum_{m^{\prime}} e^{-\theta\left(\mu_{m^{\prime}}^{s s}-M_{m^{\prime}}^{s}\right)}}$

(5) The necessary conditions associated with decision variables $f_{m p}^{r s}$ (denoting path flow by mode between each O-D pair) are:

$\frac{\partial \mathscr{\mathscr { C }}}{\partial f_{m p}^{r s}} \geq 0$

$f_{m p}^{r s} \frac{\partial \mathscr{Q}}{\partial f_{m p}^{r s}}=0$

Since

$\frac{\partial \mathscr{Q}}{\partial f_{m p}^{\prime s}}=\frac{\partial\left[\sum_{m} \sum_{l} \int_{0}^{x_{m i n}} c_{m l}(\omega) d \omega-\sum_{r s} \sum_{m} \sum_{p} \mu_{m}^{r s} f_{m p}^{s s}\right]}{\partial f_{m p}^{f s}}=\sum_{l} c_{m l} \delta_{m p}^{s i p}-\Phi_{m}^{r s}=c_{m p}^{r s}-\mu_{m}^{r s}$

Therefore, the associated necessary conditions become 
$f_{m p}^{r s} \frac{\partial \mathscr{Q}}{\partial f_{m p}^{r s}}=f_{m p}^{r s}\left(c_{m p}^{r s}-\mu_{m}^{r s}\right)=0$

$c_{m p}^{r s}-\mu_{m}^{r s} \geq 0$

Conditions (13c) and (13d) hold for each path and mode between any O-D pair in the supernetwork. If the flow on path $p$ connecting origin $r$ and destination $s$ by mode $m$ is positive $\left(f_{m p}^{r s}>0\right)$, then the corresponding path travel time is equal and minimum, i.e., $c_{m p}^{r s}=\mu_{m}^{r s}$. Otherwise, the corresponding path travel time is larger than or equal to the minimum path travel time.

From the above discussion, it is evident that optimality conditions are consistent with traveler behavior assumed for the four different travel choices, or more specifically, for generalized UE conditions.

\section{NUMERICAL EXAMPLE FOR THE COMBINED TO/TD/MC/TA MODEL WITH VARIABLE DEMAND}

With the help of the above supernetwork representation, the combined TO/TD/MC/TA model with variable demand can be regarded as an extended traffic assignment problem, for which any prevailing traffic assignment algorithm is applicable. There are several of such algorithms available in the literature, including the traditional Frank-Wolfe algorithm [13] and efficient quick-precision methods [1417]. The Frank-Wolfe algorithm is not efficient and may become obsolete for solving large-scale traffic assignment problems; however, it is simple and more suitable for pedagogical purposes.

\subsection{Frank-Wolfe Method}

Without knowing the concept of supernetworks, the Frank-Wolfe method has been used to solve various two- or three-step combined models such as the trip distribution, mode choice, and assignment [18] and the doublyconstrained, negative exponential gravity model with flowdependent travel times [19]. The Frank-Wolfe method is likely superior to a generalized Benders decomposition based algorithm [20], which is highly inefficient algorithm for solving a combined model of trip distribution and assignment, but has proven inferior to the partial linearization algorithm of Evans [12] for solving the doubly constrained combined model [19] as well as combined mode and route choice models $[21,22]$. The interested reader may refer to Boyce [23] for details.

To apply the Frank-Wolfe algorithm to the combined $\mathrm{TO} / \mathrm{TD} / \mathrm{MC} / \mathrm{TA}$ model with variable demand in Equation 8a, its linearized subproblem must be solved for the search direction. To derive the linear approximation of the objective function in Equation 8a, Taylor's series is commonly applied for a given solution $\left\{\bar{f}_{m p}^{r s}\right\}$ to the first order as follows:

$$
z[\cdot] \approx \frac{\partial z[\cdot]}{\partial f_{m p}^{r s}}\left(f_{m p}^{r s}-\bar{f}_{m p}^{r s}\right)
$$

where

$$
\begin{aligned}
& \frac{\partial z[\cdot]}{\partial f_{m p}^{r s}}=\left\{\frac{\partial z[\cdot]}{\partial x_{m l}} \frac{\partial x_{m l}}{\partial f_{m p}^{r s}}+\frac{\partial z[\cdot]}{\partial q_{m}^{r s}} \frac{\partial q_{m}^{r s}}{\partial f_{m p}^{r s}}+\frac{\partial z[\cdot]}{\partial q^{r s}} \frac{\partial q^{r s}}{\partial q_{m}^{r s}} \frac{\partial q_{m}^{r s}}{\partial f_{m p}^{r s}}+\frac{\partial z[\cdot]}{\partial q^{r}} \frac{\partial q^{r}}{\partial q^{r s}} \frac{\partial q^{r s}}{\partial q_{m}^{r s}} \frac{\partial q_{m}^{r s}}{\partial f_{m p}^{r s}}\right\} \\
& =\left\{\begin{array}{l}
\sum_{m} \sum_{l} c_{m l} \delta_{m l p}^{r s}+\sum_{r s} \sum_{m}\left(\frac{1}{\theta} \ln q_{m}^{r s}-M_{m}^{s}\right) \\
+\sum_{r s}\left(\frac{1}{\gamma} \ln q^{r s}-M^{s}\right)+\sum_{r}\left(\frac{1}{\eta} \ln q^{r}\right)-D^{-1}(q)
\end{array}\right\} \\
& =\left\{\begin{array}{l}
\sum_{r s} \sum_{m} \sum_{p} c_{m p}^{r s}+\sum_{r s} \sum_{m}\left(\frac{1}{\theta} \ln q_{m}^{r s}-M_{m}^{s}\right) \\
+\sum_{r s}\left(\frac{1}{\gamma} \ln q^{r s}-M^{s}\right)+\sum_{r}\left(\frac{1}{\eta} \ln q^{r}\right)-D^{-1}(q)
\end{array}\right\}
\end{aligned}
$$

The linearized subproblem of the original problem in Equation 8a can now be expressed as

$\min _{(\mathbf{g}, v) \in \Omega_{L}} z^{n}=\frac{\partial z[\cdot]}{\partial f_{m p}^{r s}} g_{m p}^{r s}$

$=\sum_{r s} \sum_{m} \sum_{p}\left[c_{m p}^{r s}+\left(\frac{1}{\theta} \ln q_{m}^{r s}-M_{m}^{s}\right)+\left(\frac{1}{\gamma} \ln q^{r s}-M^{s}\right)+\left(\frac{1}{\eta} \ln q^{r}\right)-D^{-1}(q)\right] g_{m p}^{r s}$

where $g_{m p}^{r s}$ denotes flows on path $p$ by mode $m$ between O-D pair $r s, \Omega_{L}$ is the feasible region associated with the linear subproblem, which is defined by the following constraints.

St.

$$
\begin{gathered}
\sum_{p} g_{m p}^{r s}=v_{m}^{r s} \quad \forall r, s, m \\
\sum_{m} v_{m}^{r s}=v^{r s} \quad \forall r, s \\
!^{r s}=!^{r} \quad \# r \\
!^{r}=! \\
!+e=?_{\max } \\
g_{m p}^{r s} !^{r s} \quad{ }^{r}, s, m, p \\
y_{m l}=\quad{ }_{r} \quad{ }_{s} g_{m p}^{r s} !_{m a p}^{r s} \quad \# m, l \\
c_{m p}^{r s}=\#{ }_{l} c_{m l} !_{m l p}^{r s} \quad r, s, m, p
\end{gathered}
$$

The meanings of Equations 14d through 14k are identical to those of Equations $6 \mathrm{~b}$ through $6 \mathrm{i}$, except the main problem variables $x, f$, and $q$ are replaced by the linear subproblem variables $y, g$, and $v$. Note that the linear subproblem in Equation $14 \mathrm{c}$ can be decomposed by origin, which not only reduces the size of the subproblem but also simplifies it as a series of one-to-all shortest path problems. Based on the derived linear subproblem, the four-step combined TO/TD/MC/TA model subject to variable demand can be easily solved by the Frank-Wolfe algorithm without any difficulty (see [13] for algorithmic steps).

\subsection{Numerical Example}

To demonstrate, we take a 21-link (indexed from 1 to 21 ) 12-node supernetwork for testing, as shown in Fig. (8). 


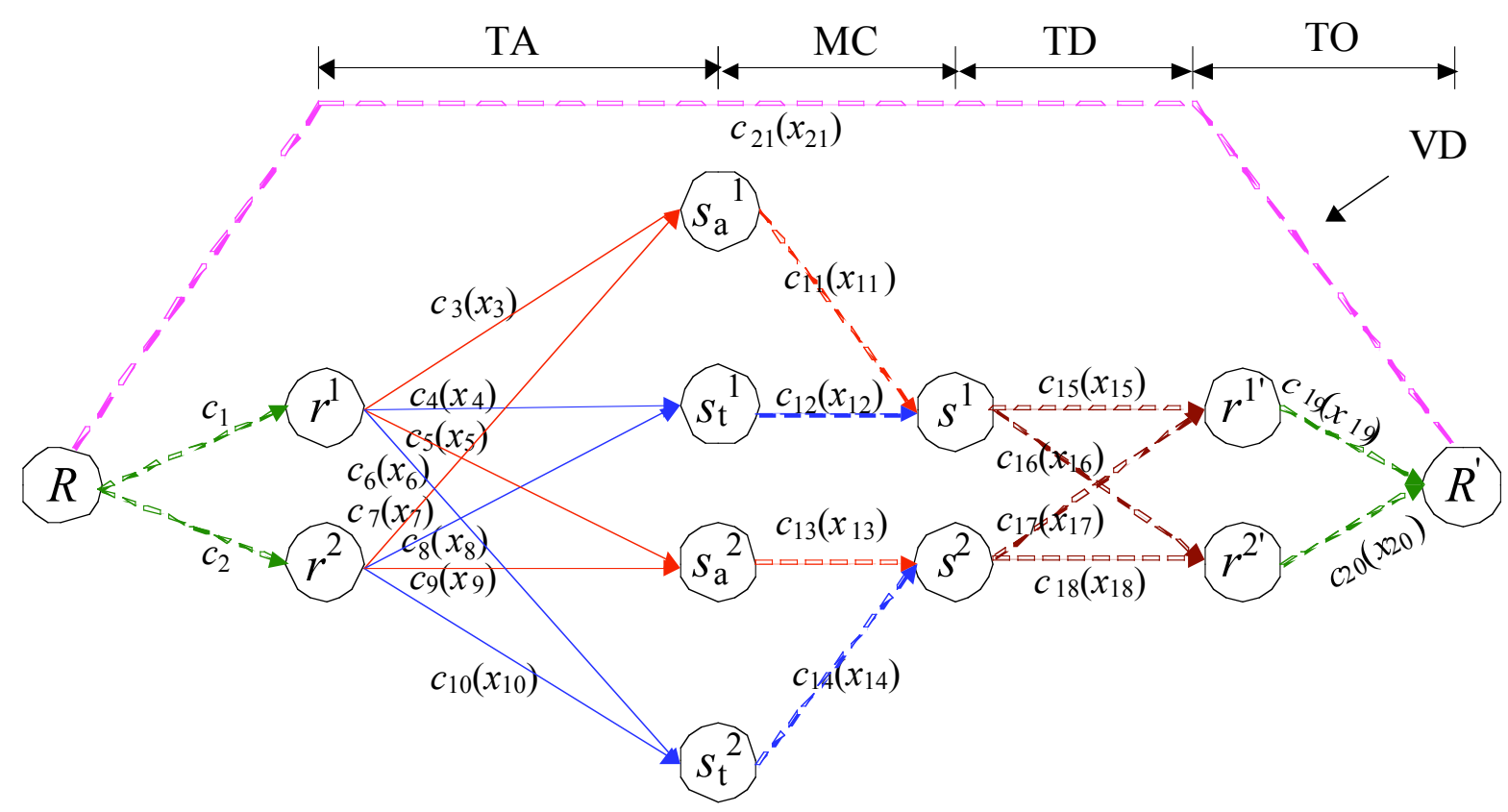

Fig. (8). Test network (supernetwork for TO/TD/MC/TA with variable demand).

The relevant data are given in Table 1:

Table 1. Input Data

\begin{tabular}{|c|c|c|}
\hline Link Cost Function & Link Cost Function & Demand \\
\hline$c_{1}=0$ & $c_{2}=0$ & \multirow{11}{*}{$\bar{q}_{\max }=100$} \\
\hline$c_{3}=0.4+0.2 x_{3}$ & $c_{7}=0.5+0.4 x_{9}$ & \\
\hline$c_{4}=8$ & $c_{8}=8.5$ & \\
\hline$c_{5}=0.5+0.4 x_{5}$ & $c_{9}=0.4+0.2 x_{7}$ & \\
\hline$c_{6}=8.5$ & $c_{10}=8$ & \\
\hline$c_{11}=\ln x_{11}$ & $c_{13}=\ln x_{13}$ & \\
\hline$c_{12}=\ln x_{12}$ & $c_{14}=\ln x_{14}$ & \\
\hline$c_{15}=\ln x_{15}$ & $c_{17}=\ln x_{17}$ & \\
\hline$c_{16}=\ln x_{16}$ & $c_{18}=\ln x_{18}$ & \\
\hline$c_{19}=\ln x_{19}$ & $c_{20}=\ln x_{20}$ & \\
\hline \multicolumn{2}{|c|}{$c_{21}=D_{r}^{-1}(\cdot)=E(\cdot)=5+2 x_{21}$} & \\
\hline
\end{tabular}

Based on the derived linear subproblem, the four-step combined model with variable demand can be solved by the Frank-Wolfe algorithm without any difficulty. The obtained results for link flows, link costs as well as dual variables are listed in Table 2:

It is observed that the obtained solution completely complies with the aforementioned optimality conditions, as follows:

\section{Trip origins}

$$
\begin{aligned}
q^{r_{1}} & =q \times \frac{e^{-\left(c_{1}+c_{3}+c_{11}+c_{15}\right)}}{e^{-\left(c_{1}+c_{3}+c_{11}+c_{15}\right)}+e^{-\left(c_{2}+c_{10}+c_{14}+c_{18}\right)}}=192.599 \times \frac{e^{-15.23508}}{e^{-15.23508}+e^{-15.23508}} \\
& =x_{15}+x_{17}=96.29936
\end{aligned}
$$

The computed results for trip origins satisfy the assumed logit formula.

2. Trip distribution

$$
\begin{aligned}
q^{r_{1} s_{1}} & =q^{r_{1}} \times \frac{e^{-\left(c_{1}+c_{3}+c_{11}\right)}}{e^{-\left(c_{1}+c_{3}+c_{11}\right)}+e^{-\left(c_{2}+c_{10}+c_{14}\right)}}=96.29936 \times \frac{e^{-11.36077}}{e^{-11.36077}+e^{-11.36077}} \\
& =x_{3}+x_{4}=48.14968
\end{aligned}
$$

The computed results for trip distribution satisfy the assumed logit formula.

3. Mode choice:

$$
\begin{aligned}
q_{\text {auto }}^{r_{1} s_{1}} & =q^{r_{1} s_{1}} \times \frac{e^{-\left(c_{1}+c_{3}\right)}}{e^{-\left(c_{1}+c_{3}\right)}+e^{-\left(c_{1}+c_{4}\right)}}=48.14968 \times \frac{e^{-7.148814}}{e^{-7.148814}+e^{-8}} \\
& =x_{3}=33.74407 \\
q_{\text {transit }}^{r_{1} s_{1}} & =q^{r_{1} s_{1}} \times \frac{e^{-\left(c_{1}+c_{4}\right)}}{e^{-\left(c_{1}+c_{3}\right)}+e^{-\left(c_{1}+c_{4}\right)}}=48.14968 \times \frac{e^{-8}}{e^{-7.148814}+e^{-8}} \\
& =x_{4}=14.40561
\end{aligned}
$$

The computed results for mode split are consistent with the logit formula.

4. Traffic assignment

At equilibrium, according to Wardrop's first principle, the travel cost on all the used paths must be equal and minimum. The computed path travel times for all the used paths are exactly the same (19.80254 units) which imply a generalized Wardrop equilibrium. 
Table 2. Computational Results

\begin{tabular}{|c|c|c|}
\hline$c_{1}=0$ & $c_{2}=0$ & $x_{1}=x_{3}+x_{4}+x_{5}+x_{6}=96.29936$ \\
\hline$c_{3}=0.4+0.2 x_{3}=7.148814$ & $c_{7}=0.5+0.4 x_{7}=13.99763$ & $x_{2}=x_{7}+x_{8}+x_{9}+x_{10}=96.29936$ \\
\hline$c_{4}=8$ & $c_{8}=8.5$ & $x_{11}=x_{3}+x_{7}=67.48814$ \\
\hline$c_{6}=8.5$ & $c_{10}=8$ & $x_{13}=x_{5}+x_{9}=67.48814$ \\
\hline$c_{11}=\ln x_{11}=4.211952$ & $c_{13}=\ln x_{13}=4.211952$ & $x_{14}=x_{6}+x_{10}=28.81123$ \\
\hline$c_{12}=\ln x_{12}=3.360766$ & $c_{14}=\ln x_{14}=3.366766$ & $x_{15}+x_{16}=x_{11}+x_{12}=48.14968 * 2$ \\
\hline$c_{19}=\ln x_{19}=4.567462$ & $c_{20}=\ln x_{20}=4.567462$ & $x_{20}=x_{16}+x_{18}=96.29936$ \\
\hline \multicolumn{2}{|c|}{$c_{21}=D_{r}^{-1}(\cdot)=E(\cdot)=5+2 x_{21}=19.80254$} & $x_{21}=\bar{q}_{\max }-x_{19}-x_{20}=7.401271$ \\
\hline
\end{tabular}

5. Variable demand

Since $c_{21}=5+2 x_{21}=5+2 \times 7.401271=19.80254$, this implies that the inverse demand is equal to the minimum travel time from all origins, i.e., $D^{-1}(q)=\mu$.

Overall, the optimality conditions are fully satisfied with this extended traffic assignment problem.

\section{CONCLUSION}

A supernetwork representation is defined as an augmented network that consists of a basic network for route choice and a virtual network for other travel choices, such as trip origins, trip distribution, or modal choice. The supernetwork representation is advantageous for addressing various combined models as an extension of the fixed demand traffic assignment problem, which can serve as a good pedagogical device and lead to new research directions. A series of supernetworks has been elaborately reviewed and criticized. In particular, mode choice dimension as well as the four-step combined model has been extensively discussed. The revised versions of the supernetworks that have been developed are useful for pedagogical purposes. Based on our discussion, two important issues arise:

1. Is the four-step sequential travel demand forecasting procedure still needed?

2. Are more efficient algorithms possible for the combined models?

For the first issue, four-step traffic demand forecasting has long been used in transportation planning. However, inconsistencies due to module interfaces often occur between the steps of travel choices. Efforts to reduce these inconsistencies have been attempted in the past [24-28]; however, problems remain, although less significant. On the other hand, centered on route choice, various combined models have appeared in the literature that is believed to be more precise than traditional four-step sequential traffic demand forecasting procedure, even with feedback. The drawback associated with combined models is that the modeling concept is difficult to interpret, at least as compared to the traffic assignment model. However, with the help of supernetwork representation, all combined models can be regarded as an extended traffic assignment problem. Thus, the four-step sequential travel demand forecasting procedure, even with feedback, may eventually become obsolete.

Regarding to the second issue, most combined models have traditionally been solved by multistage solution algorithms. Examples can be seen in the variable demand traffic assignment using the Evans algorithm [12] and the trip distribution and traffic assignment model using the double-stage algorithm [2, 29]. However, as previously discussed, the combined models can be regarded as an extension of traffic assignment and solved accordingly using any traffic assignment solution algorithm. In fact, the traffic assignment solution algorithm has made amazing progress in the last decade. Efficient algorithms such as B [30], OBA [31], TAPAS [32-35], LUCE [36] as well as the projected gradient [17] could be more efficient than traditional multistage solution algorithms. Future research will further address this issue.

Note also that some researchers argue that transportation planning field has passed the point at which each individual researchers can code their own versions of published algorithms. In this case, the recent popularity of cloud computing comes into play. Cloud computing is Internetbased computing, whereby shared resources, software and information are provided to computers and other devices on- 
demand, like a public utility. It is a paradigm shift following the mainframe and client-server shifts that preceded it. Details are abstracted from the users who no longer have need of, expertise in, or control over the technology infrastructure "in the cloud" that supports them [37]. Cloud computing describes a new supplement, consumption and delivery model for IT services based on the Internet, and it typically involves the provision of dynamically scalable and often virtualized resources as a service over the Internet [38]. It is a byproduct and consequence of the ease-of-access to remote computing sites provided by the Internet.

As a final remark, we believe that supernetwork representation can also be applied to other network applications such as economics networks [39] and supply chain network problems [40] with minor modifications. In addition, when the most complex combined TO/TD/MC/TA problem with variable demand is formulated as an extended traffic assignment model, the relationship between the sectors of urban planning and transportation is closer, and their interactions can be more precisely studied. One possible research topic in this direction is to estimate trip origins directly from traffic counts, which is analogous to the O-D estimation from the traffic assignment model. This new type of problem likely requires a bilevel model formulation and a sensitivity analysis solution technique [41], which is worth further exploration.

\section{ACKNOWLEDGEMENT}

This work was supported in part by the National Science Council, Taiwan under grant No. NSC-100-2221-E-008-093MY3. The author also thanks David Boyce, Macro Nie, and anonymous OTJ reviewers for helpful comments on drafts of the manuscript.

\section{REFERENCES}

[1] M. Beckmann, C. B. McGuire, and C. B. Winsten, Studies in the Economics of Transportation. Yale University Press: New Haven, 1956.

[2] Y. Sheffi, Urban Transportation Networks: Equilibrium Analysis with Mathematical Programming Methods. Prentice-Hall Inc: New Jersey, USA, 1985.

[3] M. Patriksson, The Traffic Assignment Problem: Models and Methods. VSP: Utrecht, 1994.

[4] N. Oppenheim, Urban Travel Demand Modeling. Wiley: New York, 1995.

[5] D. E. Boyce, L. J. LeBlanc, and K. S. Chon, "Network equilibrium models of urban location and travel choices: a retrospective survey", J. Reg. Sci., vol. 28, pp. 159-183, 1998. (JRS is now a Wiley journal)

[6] D. E. Boyce, "Network Equilibrium Models of Urban Location and Travel Choices: A New Research Agenda", In: M. Chatterji, and R. E. Kuenne, Eds., New Frontiers in Regional Science, Macmillan: New York, 1990, pp. 238-256.

[7] D. E. Boyce, "Long-Term Advances in the State of the Art of Travel Forecasting Methods", In: P. Marcotte, and S. Nguyen, Eds., Equilibrium and Advanced Transportation Modelling, Kluwer: Dordrecht, 1998, pp. 73-86.

[8] D. E. Boyce, and H. Bar-Gera, "Validation of multiclass urban travel forecasting models combining origin-destination, mode, and route choices", J. Reg. Sci., vol. 43, pp. 517-540, 2003.

[9] D. E. Boyce, and H. Bar-Gera, "Multiclass combined models for urban travel forecasting", Netw. Spat. Econ., vol. 4, pp. 115-124, 2004.

[10] J. G. Wardrop, "Some Theoretical Aspects of Road Traffic Research", In: Proceedings of Institution of Civil Engineers, Part II, no. 1, 1952, pp. 325-378.
[11] N. H. Gartner, "Optimal traffic assignment with elastic demands, a review: part II: algorithmic approaches”, Transp. Sci., vol. 14, no. 2, pp. 192-208, 1980.

[12] S. P. Evans "Derivation and analysis of some models for combining trip distribution and assignment", Transp. Res., vol. 10, pp. 37-57, 1976.

[13] M. Frank, and P. Wolfe, "An algorithm for quadratic programming”, Nav. Res. Logist. Q., vol. 3, pp. 95-110, 1956.

[14] H. Bar-Gera, "Traffic assignment by paired alternative segments (TAPAS)", Transp. Res. Part B, vol. B, pp. 1022-1046, 2010.

[15] Y. Nie, "A class of bush-based algorithms for the traffic assignment problem", Transp. Res. Part B, vol. 44, no. 1, pp. 73$89,2010$.

[16] R. B. Dial, "A path-based user-equilibrium traffic assignment algorithm that obviates path storage and enumeration", Transp. Res. part B, vol. 40, pp. 917-936, 2006.

[17] M. Florian, I. Constantin, and D. Florian, "A New Look at the Projected Gradient Method", In: CD-ROM of TRB 2009 Annual Meeting, 2009.

[18] M. Florian, and S. Nguyen, "A combined trip distribution modal split and assignment model”, Transp. Res., vol. 12, pp. 241-246, 1978.

[19] C. Frank, A Study of Alternative Approaches to Combined Trip Distribution-Assignment Modeling, PhD thesis, Regional Science, University of Pennsylvania, Philadelphia, 1978.

[20] M. Florian, S. Nguyen, and J. Ferland, "On the combined distribution-assignment of traffic”, Transp. Sci., vol. 9, pp. 43-53, 1975.

[21] M. Abdulaal, and L. J. LeBlanc, "Methods for combining modal split and equilibrium assignment models", Transp. Sci., vol. 13, pp. 292-314, 1979.

[22] L. J. LeBlanc, and K. Farhangian, "Efficient algorithms for solving elastic demand traffic assignment problems and mode-split assignment problems", Transp. Sci., vol. 15, pp. 306-317, 1981.

[23] D. E. Boyce, "Forecasting travel on congested urban transportation networks: review and prospects for network equilibrium models", Netw. Spat. Econ., vol. 7, pp. 99-128, 2007 b.

[24] H. Bar-Gera, and D. E. Boyce, "Solving the sequential procedure with feedback", In: The $6^{\text {th }}$ International Conference of Chinese Transportation Professionals, Dalian: China, 2006.

[25] D. E. Boyce, M. Lupa, and Y. Zhang, "Introducing 'feedback' into four-step travel forecasting procedure $v s$ equilibrium solution of combined model", Transp. Res. Rec., vol. 1443, pp. 65-74, 1994.

[26] D. E. Boyce, Urban Travel Forecasting Course Notes, 2007a. [online] Available: www.civil.northwestern.edu/people/boyce.html [Accessed: Dec. 28, 2007].

[27] J. D. Siegel, J. De Cea, J. E. Fernández, E. E. Rodríquez, and D. E. Boyce, "Comparisons of urban travel forecasts prepared with the sequential procedure and a combined model", Netw. Spat. Econ., vol. 6, pp. 135-148, 2006.

[28] D. E. Boyce, C. R. O'Neill, and W. Scherr, "New results on solving the sequential travel forecasting procedure with feedback", In: the $17^{\text {th }}$ PTV Vision User Group Meeting, Berlin, 2007.

[29] H. K. Chen, and Y. C. Chen, "Comparisons of the Frank-Wolfe and Evans methods for the doubly constrained entropy distribution/ assignment problem", J. Eastern Asia Soc. Transp. Stud., vol. 3, no. 1, pp. 261-276, 1999.

[30] R. B. Dial, "A path-based user-equilibrium traffic assignment algorithm that obviates path storage and enumeration", Transp. Res. part B, vol. 40, pp. 917-936, 2006.

[31] H. Bar-Gera, "Origin-based algorithm for the traffic assignment problem”, Transp. Sci., vol. 36, no. 4, pp. 398-417, 2002.

[32] H. Bar-Gera, "Traffic assignment by paired alternative segments (TAPAS)", Transp. Res. Part B, vol. 44, pp. 1022-1046, 2010.

[33] H. Bar-Gera, and D. E. Boyce, "Some Amazing Properties of Road Traffic Network Equilibria”, In: T. L. Friesz, Ed., Network Science, Nonlinear Science and Infrastructure Systems, Springer: Berlin, 2007, pp. 305-335.

[34] D. E. Boyce, Y. Nie, H. Bar-Gera, Y. Liu, and Y. Hu "Consistent route flows and the condition of proportionality", In: the 2010 Annual Meeting of the Transportation Research Board, Washington, DC, 2010a.

[35] D. E. Boyce, Y. Nie, H. Bar-Gera, Y. Liu, and Y. Hu, "Field Test of a Method for Finding Consistent Route Flows and MultipleClass Link Flows", Draft report to the Federal Highway Administration, Washington, DC, 2010b. 
[36] G. Gentile, "Linear user cost equilibrium: a new algorithm for traffic assignment", Submitted to Transp. Res. Part B, 2009.

[37] K. Danielson, Distinguishing Cloud Computing from Utility Computing, SaaS Week, 2008. [online] Available: www.ebizq.net/blogs/ saasweek/2008/03/distinguishing_cloud_computing. [Accessed: March 17, 2010].

[38] E. Knorr, and G. Gruman, What Cloud Computing Really Means, InfoWorld, 2008. [online]: www.infoworld.com/d/cloud-computing/ what-cloud-computing-really-means-031, [Accessed: March 17, 2010].

[39] A. Nagurney, Network Economics: A Variational Inequality Approach, Kluwer Academic Publishers: Massachusetts, 1993.

[40] H. K. Chen, and H. W. Chou, "Supply chain network equilibrium problem with capacity constraints", Pap. Reg. Sci., vol. 87, no. 4, pp. 605-621, 2008.

[41] R. L. Tobin, and T. Friesz, "Sensitivity analysis for equilibrium network flow", Transp. Sci., vol. 22, no. 4, pp. 242-250, 1988.

Received: October 5, 2011

Revised: October 25, 2011

Accepted: November 3, 2011

(C) Huey-Kuo Chen; Licensee Bentham Open.

This is an open access article licensed under the terms of the Creative Commons Attribution Non-Commercial License (http://creativecommons.org/licenses/by-nc/

$3.0 /$ ) which permits unrestricted, non-commercial use, distribution and reproduction in any medium, provided the work is properly cited. 